planning; plans; responses; testing Prehosp Disast Med 2002;17(s2):s74-75.

\section{High School Students Assist a Hospital in Mass Casualty Events}

Nurit Fertel, $R N, B A ;$ Bianca Lederman, $M D$

Tel Aviv Sourasky Medical Center (TASMC), Israel

Objective: The Israeli Medical System is committed to administering optimal medical services to casualties during conventional and nonconventional mass casualty events (MCE). The operation of the hospital in such events requires the expansion of facilities, predesignation of admitting sites, and operation of special roles.

Methods: Ancillary and voluntary teams are needed. This hospital initiated a model cooperation program with a neighboring high school. Approximately 300 students from the 10 th to 12 th grades are integrated into the hospital personnel during a MCE. The students serve as stretcher-bearers and carry out other necessary tasks. Once a year, the students participate in a training program and drills.

Results: As a result of the ongoing relationship over the last 10 years between the hospital management and the director of the school, the students are available to help every moment, throughout the whole year. This project has been supported by the Municipality Emergency law and recognized by the army and the Ministry of Health.

Conclusion: This unique model has many benefits to the community and to the hospital, but mainly for the students themselves. The most important education a student can receive is the ability to contribute to others.

Keywords: auxillary personnel; drills; high schools; hospital; management; mass casualty events; students; training

Prehosp Disast Med 2002;17(s2):s75.

\section{The British Red Cross Society \\ Dr. Judith M. Fisher, FRCGP, FFAEM, FIMCEd}

Chief Medical Advisor, The British Red Cross Society

The British Red Cross Society (BRCS) is a nationwide organization of volunteers who provide aid and assistance in a wide range of spheres. Although recognized for firstaid skills, the association provides many other caring services such as escorting patients, welfare services, counseling, and a unique camouflage service, provided by 225 practitioners. Started as an aid to scarred military casualties in the Second World War, it now sees more than 6,000 patients each year.

The BRCS has an active International Division, which is part of the largest humanitarian organization in the world. As part of the Federation of Red Cross and Red Crescent Societies (175 national societies) and as a partner of the International Committee of the Red Cross (ICRC), it is a key partner in the Strategy 2010 - "To Improve the Lives of Vulnerable People by Mobilizing the Power of Humanity." The BRCS has teams working in many countries throughout the world.

This paper examines the diverse work of the organization, and how it can work together with the World Association for Disaster and Emergency Medicine to work toward the goals of Strategy 2010.

Keywords: assistance; British Red Cross Society (BRCS); first aid skills;
International Committee of the Red Cross (ICRC); Strategy 2010; World Association for Disaster and Emergency Medicine (WADEM) Prehosp Disast Med 2002;17(s2):s75.

\section{Requirements Generation for Individual Protective Equipment}

Meave Garigan

Johns Hopkins University, Baltimore, Maryland, USA

Historically, the development of equipment that protects individuals from nuclear, biological, and chemical (NBC) threats has been a military endeavor. Although civilian versions of similar equipment have long been available, the technology has been driven by military requirements. However, NBC and industrial hazards are a growing public health concern, and the civilian need for individual protective equipment (IPE) soon will outstrip military demands.

Although development efforts rarely have worked in tandem, civilian and military IPE needs have a great deal in common. We have identified key requirements that apply to the development of both civilian and military IPE, emphasizing low-cost, low-maintenance, easy-to-use, reliable solutions with the capability for performance upgrades. This emphasis enables civilian use, including use in less industrialized areas, where training and material support may be nonexistent. The capability for performance upgrades allows enhancements for rigorous military use. For instance, the current United States military project to develop a disposable NBC hood will result in two versions, a civilian-style hood for escape and an upgraded model that will allow users to conduct military missions. Overarching requirements will conserve resources during design and manufacture, and will aid in the effort to create industry standards.

Increasing accessibility to quality, low-cost IPE will help prevent mass casualties during terrorist or industrial disasters, whether they occur in New York City or Bhopal. By combining common goals and rethinking approaches to equipment design, we will better serve both civilian and military populations.

Keywords: accessibility; biological; chemical; design; hazards; individual protective equipment; military; nuclear; requirements; standards Prehosp Disast Med 2002;17(s2):s75.

\section{Respiratory Sinus Arrhythmia Feedback for Enhanced Parasympathetic Responses in Clients Diagnosed with Post-Traumatic Stress Disorder and Anxiety: A Case Study \\ Phillip Fourie}

Assistant Professor, School for Health Sciences, Bond University, Queensland, Australia

We live in a world of hazards, with increasing terrorism activities, natural disasters, and personal trauma. Post-traumatic stress disorder (PTSD) and anxiety-related disorders are part of modern society. The anxiety response is one of the mechanisms the body uses to help the mind recognize danger and minimize it. As with most psychological disorders, it is not the presence of anxiety that creates problems, it is more about how severe it is, how the individual copes with it, and how much it disrupts the individual's ability to 
deal with "life demands."

The two major treatment modalities for anxiety-based conditions are medical (physiological) and psychological. Medical treatment, consisting of pharmaceutical products such as antidepressants and anxiolytics, does not address the problem directly, but concentrates on the reduction of anxiety and affective symptoms. This case study focuses on a behavioral intervention. Most clients suffering from PTSD and anxiety-related disorders have an imbalance of the autonomic nervous response, with an increased function of the sympathetic nervous system, and a decreased function of the parasympathetic nervous system. Respiration sinus arrhythmia (RSA) is a noninvasive measure of parasympathetic tone that has been related to emotion regulatory capacity. RSA refers to the cyclical variation in heart rate associated with respiration, which can be modified by rate and depth of breathing. High RSA levels among depressed or anxious individuals predict more pernicious course of illness and a poor prognosis of recovery than lower RSA levels.

Modern signal processing such as Bioview Series IV provides a means of measuring and analyzing heart rate and RSA, so to permit a quantitative, noninvasive method of assessing the autonomic nervous system's effect on the body.

This case study demonstrates the effects of respirationbased training as a modality to increase RSA, and therefore, the influence on the activation of the parasympathetic nervous system.

Keywords: anxiety-related disorders; illness, course of; parasympathetic tone; post-traumatic stress disorder (PTSD); respiratory sinus arrhythmia (RSA); training, respiratory-based; treatment

Prehosp Disast Med 2002;17(s2):s75-76.

\section{Comparison of Mainz Emergency Evaluation System (MEES) and Revised Trauma Score (RTS) for Prediction of Mortality of Trauma Patients \\ S. Grmec; ${ }^{1}$ T. Schaubach, ${ }^{1}$ E. Stok, ${ }^{2}$ J. Ferk ${ }^{3}$ \\ 1. Center for Emergency Medicine, Maribor, Slovenia \\ 2. Ministry for Health, Emergency Medicine Project, Ljubljana, Slovenia \\ 3. Teaching Hospital, Maribor, Slovenia}

Introduction: There are numerous prehospital, descriptive scoring systems, and it is uncertain whether they are efficient in assessing of the severity of injury, and whether they have a prognostic role in the estimation of the injury outcome. The purpose of this study was to assess the value of the Mainz Emergency Evaluation System (MEES) and the Revised Trauma Score (RTS) in predicting the outcome of trauma patients.

Method: In a prehospital setting, values for MEES and RTS were measured for each trauma patient. This study was undertaken over four years (January 1998 to July 2002) and included 286 consecutive patients (188 male and 98 female) hospitalized for trauma (polytrauma, multitrauma, trauma with shock or/and coma). Their ages ranged from 16 to 81 years $(45.3 \pm 169)$. Patients younger than 16 years of age were not included. Sensitivity, specificity, and correct prediction of outcome were assessed using the two severity scores. The best cut-off point in each scoring system was determined using the Youden index. The difference in Youden index was calculated using the $\mathrm{Z}$-score. For each score, the receiver operating characteristic (ROC) curve was obtained. The difference in ROC was calculated using the $Z$-score. A $p$-value of $<0.05$ was considered statistically significant.

Results: For prediction of mortality, the best cut-off points were 18 for MEES and 7 for RTS. The best cut-offs for the Youden index were 0.63 for MEES and 0.60 for RTS. The correct prediction of outcome was achieved in $79.6 \%$ for MEES and $75.8 \%$ for RTS. The area under the ROC curve was $0.83 \pm 0.04$ for MEES and $0.79 \pm 0.06$ for RTS. There were no statistically significant differences among MEES and RTS in terms of correct prediction of outcome, Youden index, or area under ROC curve.

Conclusions: Use of the MEES for prediction of mortality in patients with trauma is equal to use of the RTS, and in a prehospital setting, it is not necessary to combine it with the RTS. The MEES also is valuable in predicting outcome for patients with other diseases. Because of this, the MEES provides a good scoring system for quick evaluation of the emergency status as well as for predicting the outcome of patients in a prehospital setting, especially in mass casualty situations, where the combination of trauma and other diseases is expected (e.g., combination of trauma and poisoning).

Keywords: Mainz Emergency Evaluation System (MEES); mortality; outcome; prediction; prognosis; Revised Trauma Score (RTS); trauma; Youden index Prebosp Disast Med 2002;17(s2):s76.

\section{Characteristics of Biological Terrorism and Managing Measures \\ Prof. Sbusen Guo}

Medical Service Department of the Logistics Command

Academy of Chinese People's Liberation Army, Beijing, China

Since the events of 11 September 2001, more and more attention has been paid to biological terrorism. Characteristics of biological terrorism include: (1) Potentiality; there are more than 1,500 bacteria banks in the world, and an uncountable number of institutes from which terrorists could get biological agents. (2) There have been scattered occurrences, not only in an area, but also in time. (3) Concealed easily, biological agents can be put into food and other materials. (4) Suddenness of appearance make it difficult to forecast. (5) Often, it occurs with another kind of terrorism. (6) Easily hidden with other materials to create panic.

Before an incident occurs, we should prepare regulations and programs, study technology and skills, store up materials, and train personnel. After the incident occurs, we should identify the danger, identify the biological agent, forecast the diseases that may result, evaluate the number of patients and areas into which it is likely to spread, put forward measures to reduce the harm, prevent people from exposure, treat patients, and assess the effectiveness of managing measures and adjust them properly.

Keywords: bacteria banks; characteristics; forecast; prediction; preparation; regulations; responses; terrorism, biological Prebosp Disast Med 2002;17(s2):s76. 